\title{
CORRECTION TO: CLUSTER ALGEBRAS FROM SURFACES AND EXTENDED AFFINE WEYL GROUPS
}

ANNA FELIKSON*

Department of

Mathematical Sciences

Durham University

Science Laboratories, South Road

Durham, DH1 3LE, UK

anna.felikson@durham.ac.uk

MICHAEL SHAPIRO

Department of

Mathematics

Michigan State University

East Lansing, MI 48824, USA

and

National Research University

Higher School of Economics, Russia

mshapiro@math.msu.edu
JOHN W. LAWSON

Codeplay Software Ltd

3 Lady Lawson Street

Edinburgh

EH3 9DR, UK

john@codeplay.com

\section{PAVEL TUMARKIN}

\author{
Department of \\ Mathematical Sciences \\ Durham University \\ Science Laboratories, South Road \\ Durham, DH1 3LE, UK \\ pavel.tumarkin@durham.ac.uk
}

Correction to: Transformation Groups 2021, 26(2): pp. 501-535

https://doi.org/10.1007/s00031-021-09647-y

The original version of this article unfortunately contained some mistakes in web displays of author order.

This has been corrected to read Anna Felikson, John W. Lawson, Michael Shapiro, Pavel Tumarkin.

DOI: $10.1007 /$ s00031-021-09663-y

Corresponding Author: Pavel Tumarkin, e-mail: pavel.tumarkin@durham.ac.uk 